\title{
An Evaluation of Maturity Estimates Derived from Two Different Sampling Schemes: Are the Observed Changes Fact or Artifact?
}

\author{
S. E. Wigley, J. M. Burnett, and P. J. Rago \\ National Marine Fisheries Service \\ Woods Hole, MA 02543 USA
}

\begin{abstract}
Biological sampling of fish during research vessel survey cruises is often conducted using length-stratified age sampling schemes. Maturity-at-length or age may be affected by changes in sampling protocols, and may potentially result in biased estimates of maturation patterns. In such cases, these estimates may be artifacts of the sampling activities and not accurate estimators of stock dynamics.
\end{abstract}

In 1992, the Northeast Fisheries Science Center (NEFSC) modified the length-stratified age sampling protocol used during semi-annual research vessel bottom trawl surveys. While demonstrable gains in the precision of age-length keys for many species have been obtained with the new protocols, no systematic evaluation of the effects of the sampling protocol change upon maturity-at-length or age has been performed.

In this study, potential effects of changes in sampling protocol were assessed for two groundfish species, haddock (Melanogrammus aeglefinus) and American plaice (Hippoglossoides platessoides). Over the critical size range of maturity for both species, significantly different length frequency sampling was obtained from each sampling scheme during the 10-year study period. Analyses for American plaice were complicated by the sexually dimorphic growth. Simulation techniques were employed to assess whether the observed changes in maturity estimates were a result of the changed sampling protocols. The simulations suggested that these changes were real phenomena and not artifacts caused by changes in sampling.

Key words: length-stratified sampling, maturity-at-length

\section{Introduction}

Biological sampling of fish during research vessel surveys is often accomplished by using length-stratified sampling schemes. These schemes are designed to derive satisfactory relationships between length and age, but may not always produce data suitable for estimation of other population parameters such as growth or maturation rates (Armstrong and Ilardia, MS 1986; Anon., MS 1994; Morgan and Hoenig, 1997). Moreover, changes in protocols during a sampling time series may potentially result in biased estimates of population patterns. In such cases, these estimates may be artifacts of the sampling activities and not accurate estimators of stock dynamics.

In 1992, the Northeast Fisheries Science Center (NEFSC) modified the length-stratified sam- pling protocol used during research vessel bottom trawl surveys which have been conducted since 1963. Prior to 1992, target numbers of fish to be sampled within broad (several $\mathrm{cm}$ ) length strata were specified for each 6-hour watch of the survey cruise. In 1992, the sampling procedure was revised to sampling one fish at each $\mathrm{cm}$ interval per tow (Table 1). While demonstrable gains in the precision of age-length keys for various species have been obtained (NEFSC, MS 1992) with the new protocols, no systematic evaluation of the effects of the sampling change on maturity-at-length or age has been performed.

In this study, the effects of the 1992 change in sampling protocol on estimated maturity parameters for two groundfish species, haddock (Melanogrammus aeglefinus) and American plaice (Hippoglossoides platessoides) are assessed. A 
TABLE 1. Sampling protocols for length-stratified sampling of haddock and American plaice used during Northeast Fisheries Science Center research vessel bottom trawl surveys, 1963-91 and 1992-present.

\begin{tabular}{llrl}
\hline \hline & \multicolumn{2}{c}{ Pre-1992 } & Post-1992 \\
& per 6 hour watch & per tow \\
Haddock & $<8 \mathrm{~cm}$ & $10 \mathrm{fish}$ & 1 fish per cm \\
& $9-25 \mathrm{~cm}$ & $5 \mathrm{fish}$ & \\
& $26-50 \mathrm{~cm}$ & $15 \mathrm{fish}$ & \\
& $>50 \mathrm{~cm}$ & $45 \mathrm{fish}$ & \\
& & & \\
American plaice & $<20 \mathrm{~cm}$ & all fish & 1 fish per cm \\
& $21-30 \mathrm{~cm}$ & $5 \mathrm{fish}$ & \\
& $31-40 \mathrm{~cm}$ & $25 \mathrm{fish}$ & \\
& $>40 \mathrm{~cm}$ & $20 \mathrm{fish}$ & \\
\hline
\end{tabular}

Monte Carlo-type simulation model was developed which allowed re-sampling of survey catches of these species prior to 1992 and after 1992 using both sampling schemes. The effects of the two sampling schemes were then compared using the simulated estimates of maturity-at-length versus those obtained empirically.

\section{Methods}

NEFSC research vessel bottom trawl survey data from 1987-96 were examined for cases in which changes in length-stratified age sampling in 1992 might have resulted in biased maturity estimates for certain species. The evaluation involved two considerations: 1) situations in which length strata in the pre-1992 sampling scheme were relatively broad, and 2) of these, length strata which encompassed the critical size range associated with the onset of maturation. Two species, Georges Bank haddock and American plaice were selected for examination. Georges Bank haddock was chosen because a pre-1992 length stratum of $26-50 \mathrm{~cm}$ was relatively wide and encompassed fish sizes for which observed proportions mature ranged from 30 to $100 \%$ (O'Brien et al., 1993). American plaice was selected because it exhibits sexually dimorphic growth and maturation rates; males maturing at a smaller size and younger age than females (O'Brien et al., 1993).

Size at maturation was evaluated using data collected prior and close to the time of spawning (Halliday, 1987). Maturity data for both species were derived from NEFSC spring research vessel bottom trawl surveys. The data were pooled into two five-year blocks, 1987-91 and 1992-96 (representing equal number of years of data collection under each sampling scheme). Probit analyses (Finney, 1971) of proportions mature at length were used to derive maturity ogives and associated $95 \%$ confidence intervals for female haddock and male and female American plaice for each study period.

To evaluate whether the 1992 change in sampling protocol resulted in differing sampling distributions, the frequency distribution of fish sampled at each centimeter in the two five-year blocks of pooled data were compared using Chisquare analysis (Sokal and Rohlf, 1981). The expected distribution of the total numbers caught at length during 1992-96 period was derived by multiplying the observed proportions sampled at length in 1987-91 by the estimated total numbers caught at length. The null hypothesis in this procedure is that the realized distribution sampled in 1992-96 was equivalent to what would have been sampled under the 1987-91 sampling protocol.

A Monte Carlo-type simulation model was constructed to evaluate the effects of the sampling protocols on estimated maturity parameters for each species. Numbers of fish at length were drawn from each of the pooled five-year blocks of catch-atlength data using the sampling protocol associated with the opposite five-year period (i.e. alternative sampling). The maturity status of each sampled fish was considered as the outcome of a Bernoulli trial in which the underlying probability of being mature was derived from a probit analysis of the original sampled data. Probit analysis was applied to each simulated data set and the process was repeated 
500 times for each comparison. The primary statistics of interest were the estimated lengths at which $10,25,50,75$, and $90 \%$ of the population was mature (i.e. $L_{10}, L_{25}, L_{50}, L_{75}$ and $L_{90}$ ), respectively. Frequency distributions and associated median values of maturity estimates corresponding to $L_{10}, L_{25}$, $L_{50}, L_{75}$ and $L_{90}$ were derived. Baseline maturity estimates were produced for each five-year period by repeating the procedure outlined above using the sampling design that actually existed during the period. Median values of maturity estimates obtained from alternative sampling were then evaluated with respect to the $80 \%$ confidence intervals of the corresponding estimates derived from the baseline runs.

The simulation approach applied to evaluate effects of sampling protocol is summarized below:

\begin{tabular}{lcc}
\hline & \multicolumn{2}{c}{ Sampling scheme } \\
\cline { 2 - 3 } Maturity data & $1987-91$ & $1992-96$ \\
\hline $1987-91$ & Baseline & Alternative \\
$1992-96$ & Alternative & Baseline \\
\hline
\end{tabular}

where maturity data are the proportion mature at length and the sampling scheme is the number of fish sampled at length in each time block.

\section{Results}

Sampling intensity for haddock and American plaice during the 1987-91 and 1992-96 periods is summarized in Table 2. The percentage of survey stations sampled were comparable under both sampling schemes, and similar numbers of fish sampled in both periods for each species.

TABLE 2. Sampling intensity of haddock and American plaice for five-year time blocks prior to and subsequent to the change in NEFSC research vessel bottom trawl survey length-stratified age sampling protocol in 1992.

\begin{tabular}{|c|c|c|c|c|}
\hline & \multicolumn{2}{|c|}{ Number of stations } & \multicolumn{2}{|c|}{ Number of fish } \\
\hline & Caught & Sampled & Caught & Sampled \\
\hline \multicolumn{5}{|c|}{ Georges Bank Haddock } \\
\hline 1987 & 23 & 20 & 409 & 96 \\
\hline 1988 & 26 & 21 & 249 & 149 \\
\hline 1989 & 31 & 30 & 588 & 251 \\
\hline 1990 & 23 & 23 & 723 & 246 \\
\hline 1991 & 21 & 20 & 322 & 184 \\
\hline & \multicolumn{2}{|c|}{$92 \%$ coverage } & \multicolumn{2}{|r|}{926 fish } \\
\hline 1992 & 23 & 23 & 139 & 105 \\
\hline 1993 & 25 & 25 & 265 & 153 \\
\hline 1994 & 22 & 22 & 933 & 124 \\
\hline 1995 & 19 & 19 & 796 & 208 \\
\hline \multirow[t]{2}{*}{1996} & 28 & 28 & 1827 & 343 \\
\hline & \multicolumn{2}{|c|}{$100 \%$ coverage } & \multicolumn{2}{|r|}{933 fish } \\
\hline \multicolumn{5}{|l|}{ American plaice } \\
\hline 1987 & 49 & 42 & 523 & 349 \\
\hline 1988 & 51 & 50 & 513 & 360 \\
\hline 1989 & 52 & 43 & 680 & 367 \\
\hline 1990 & 57 & 53 & 818 & 384 \\
\hline 1991 & 54 & 50 & 874 & 486 \\
\hline & \multicolumn{2}{|c|}{$90 \%$ coverage } & \multicolumn{2}{|r|}{1946 fish } \\
\hline 1992 & 61 & 60 & 567 & 383 \\
\hline 1993 & 58 & 58 & 649 & 363 \\
\hline 1994 & 65 & 63 & 814 & 376 \\
\hline 1995 & 67 & 65 & 1203 & 514 \\
\hline \multirow{2}{*}{1996} & 65 & 62 & 954 & 380 \\
\hline & \multicolumn{2}{|c|}{$97 \%$ coverage } & \multicolumn{2}{|r|}{2016 fish } \\
\hline
\end{tabular}


Significantly different maturity ogives between the two five-year periods were obtained for female Georges Bank haddock and for both sexes of American plaice (Fig. 1 and 2). For haddock, maturation

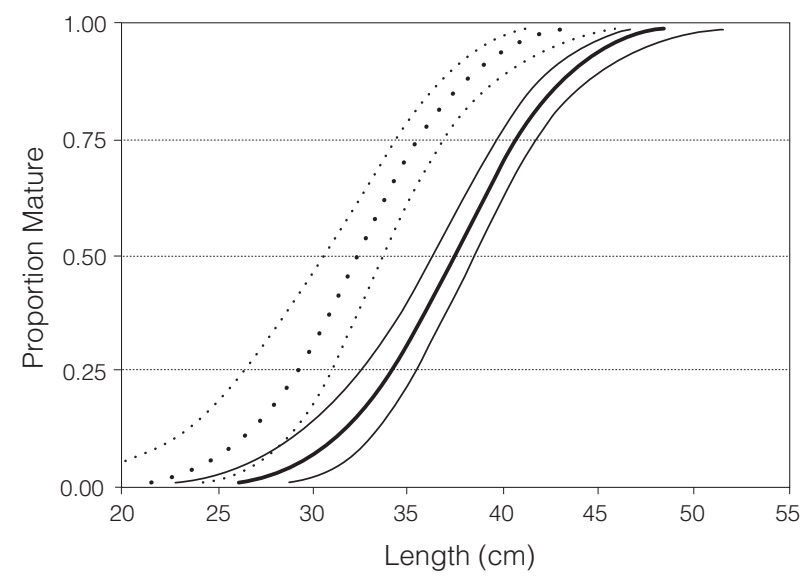

Fig. 1. Maturity ogives of proportions mature at length (cm, and associated 95\% Confidence Intervals) for female Georges Bank haddock derived from probit analyses of maturity observations collected during NEFSC spring research vessel bottom trawl surveys in 1987-91 (dotted line) and 1992-96 (solid line).

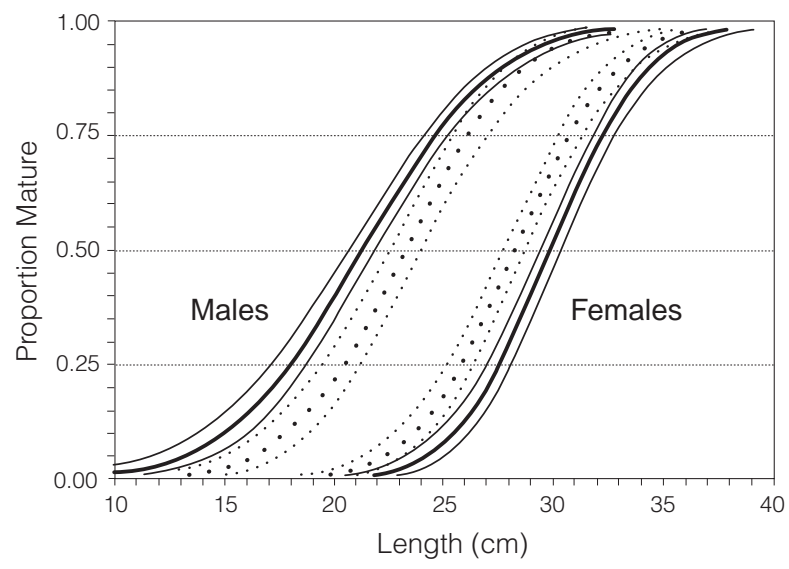

Fig. 2. Maturity ogives of proportions mature at length $(\mathrm{cm}$, and associated $95 \%$ Confidence Intervals) for male and female American plaice derived from probit analyses of maturity observations collected during NEFSC spring research vessel bottom trawl surveys in 198791 (dotted line) and 1992-96 (solid line). occurred at smaller sizes in the 1987-91 period although at about the same rate (i.e., slope of the ogive). Estimates of $L_{50}$, the median length at maturity, was $32.5 \mathrm{~cm}$ for female Georges Bank haddock during 1987-91 and $37.5 \mathrm{~cm}$ during 1992-96. For American plaice, maturation of males occurred at smaller sizes in the 1992-96 period, but females matured at greater sizes for that same period. Values of $L_{50}$ for male plaice were $23.2 \mathrm{~cm}$ and 21.3 $\mathrm{cm}$ for the 1987-91 and 1992-96 periods respectively, and $28.2 \mathrm{~cm}$ and $29.8 \mathrm{~cm}$, respectively, for female plaice.

The empirical pooled length frequency catch and sampling distribution for each five-year period are presented in Fig. 3 for haddock and Fig. 4 for American plaice. Significant differences were obtained in the sampled distributions between periods for each species (for haddock, $X^{2}=725.84$, $p<0.01,54 \mathrm{df}$; for American plaice, $X^{2}=191.97$, $p<0.01,48 \mathrm{df})$.

Figure 5a-d provides an example of the simulation results for female Georges Bank haddock. The frequency distributions and medians of estimates of $L_{10}$ obtained from the 500 probit analyses are plotted for each of the four simulation scenarios: a) alternative sampling of 1987-91 data; b) baseline run for 1987-91; c) alternative sampling of 199296 data; and d) baseline run for 1992-96. Median estimates from the baseline runs at each level $L_{10^{-}}$ $L_{90}$ were within $0.1 \mathrm{~cm}$ of the corresponding values from the original maturity ogives for each species in each time period.

Figures 6-8 present simulation estimates of $L_{10}-L_{90}$ for Georges Bank haddock and male and female American plaice, respectively. For haddock (Fig. 6), all median values of proportions mature at length generated from the alternative sampling simulations lie within the $80 \%$ confidence interval of the baseline runs. Median values of alternative sampling become more similar to baseline values at increasing levels of proportions mature at length. Variability in the distribution of estimates for both alternative sampling and baseline simulations was greatest at the upper and lower tails (i.e. $L_{10}$ and $L_{90}$ ) than for $L_{50}$. In no instance did the median maturity estimates from the 1987-91 period overlap with the $80 \%$ confidence intervals from the 


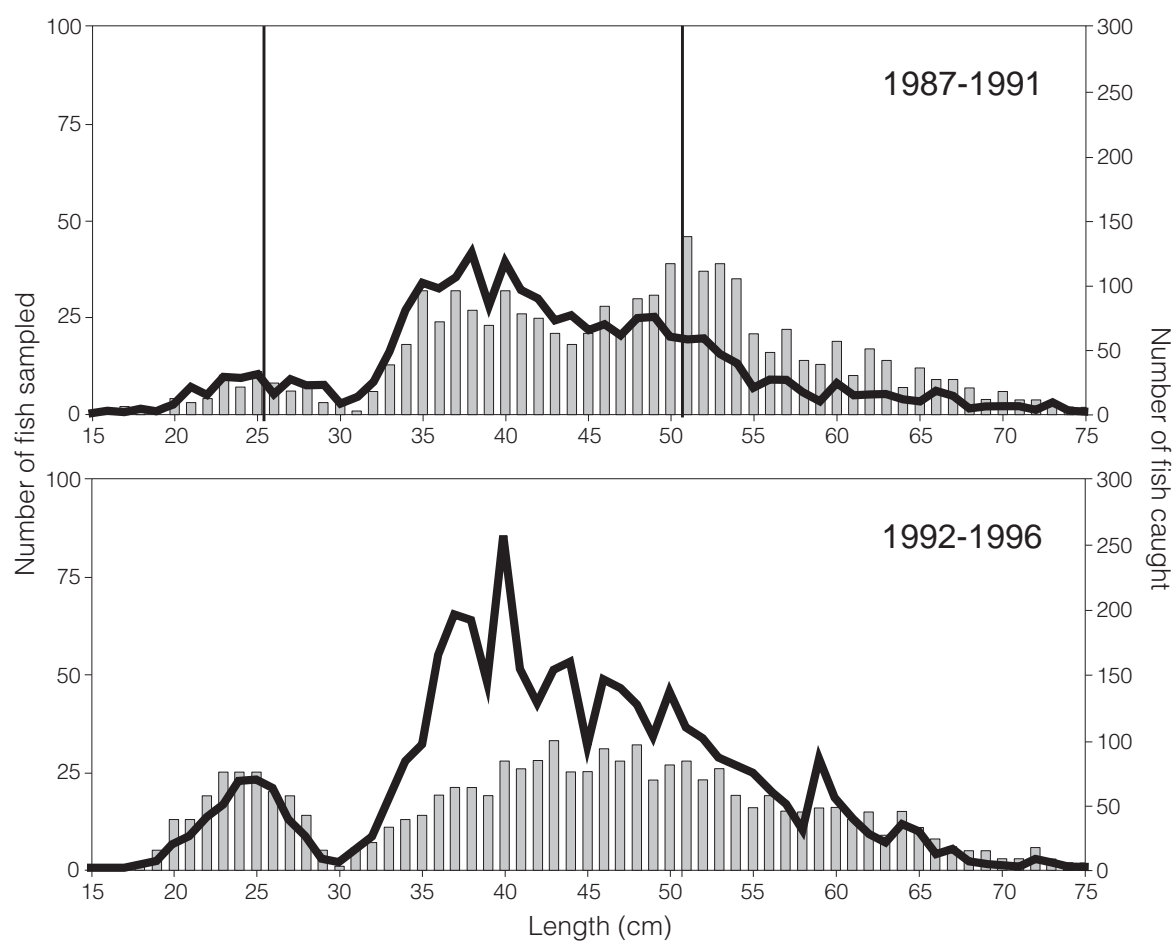

Fig. 3. Length frequency distributions of the total catch (solid lines) and sampled catch (bars) of Georges Bank haddock during NEFSC spring research vessel bottom trawl surveys in 1987-91 and 1992-96. Vertical lines represent length strata in the 1987-91 sampling period (see Table 1).

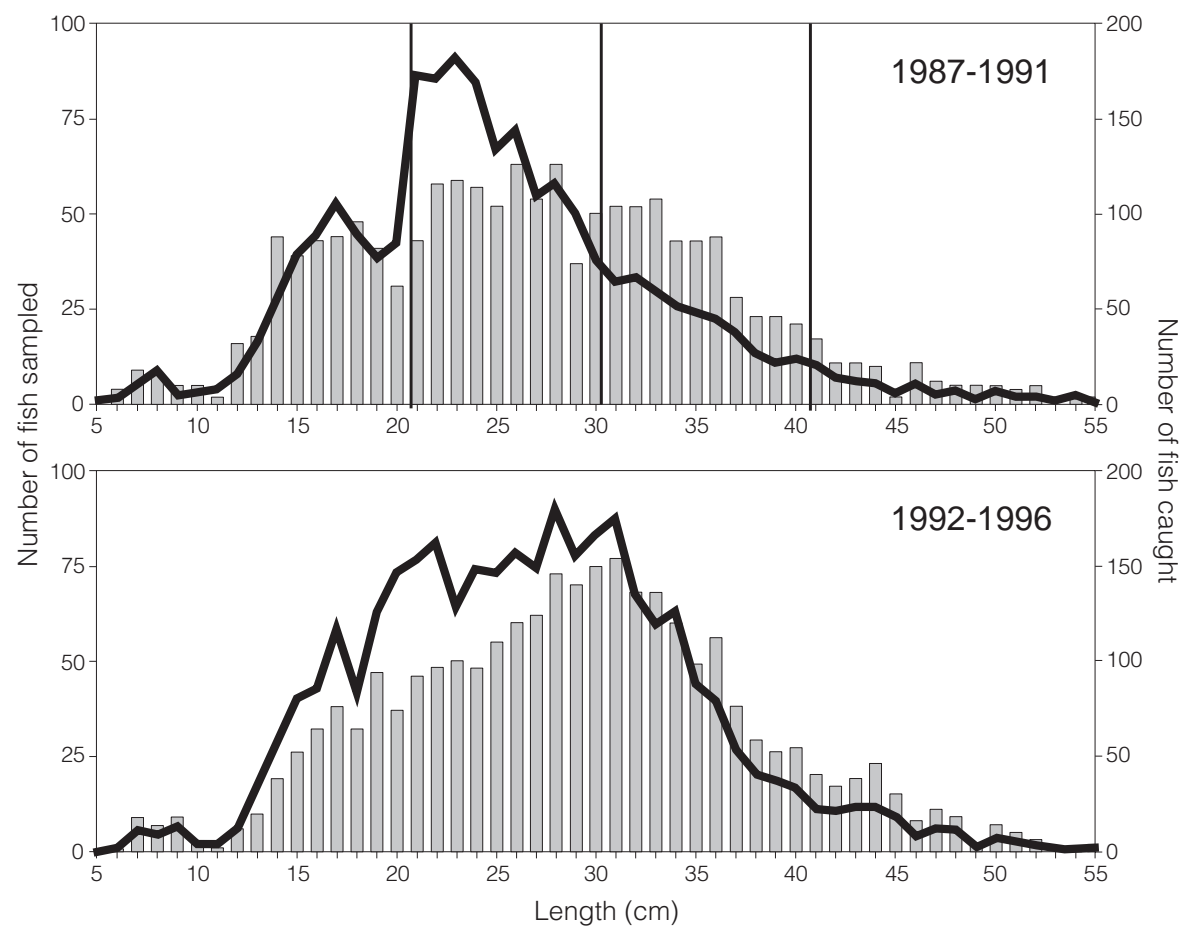

Fig. 4. Length frequency distributions of the total catch (solid lines) and sampled catch (bars) of American plaice during NEFSC spring research vessel bottom trawl surveys in 1987-91 and 1992-96.Vertical lines represent length strata in the 1987-91 sampling period (see Table 1). 


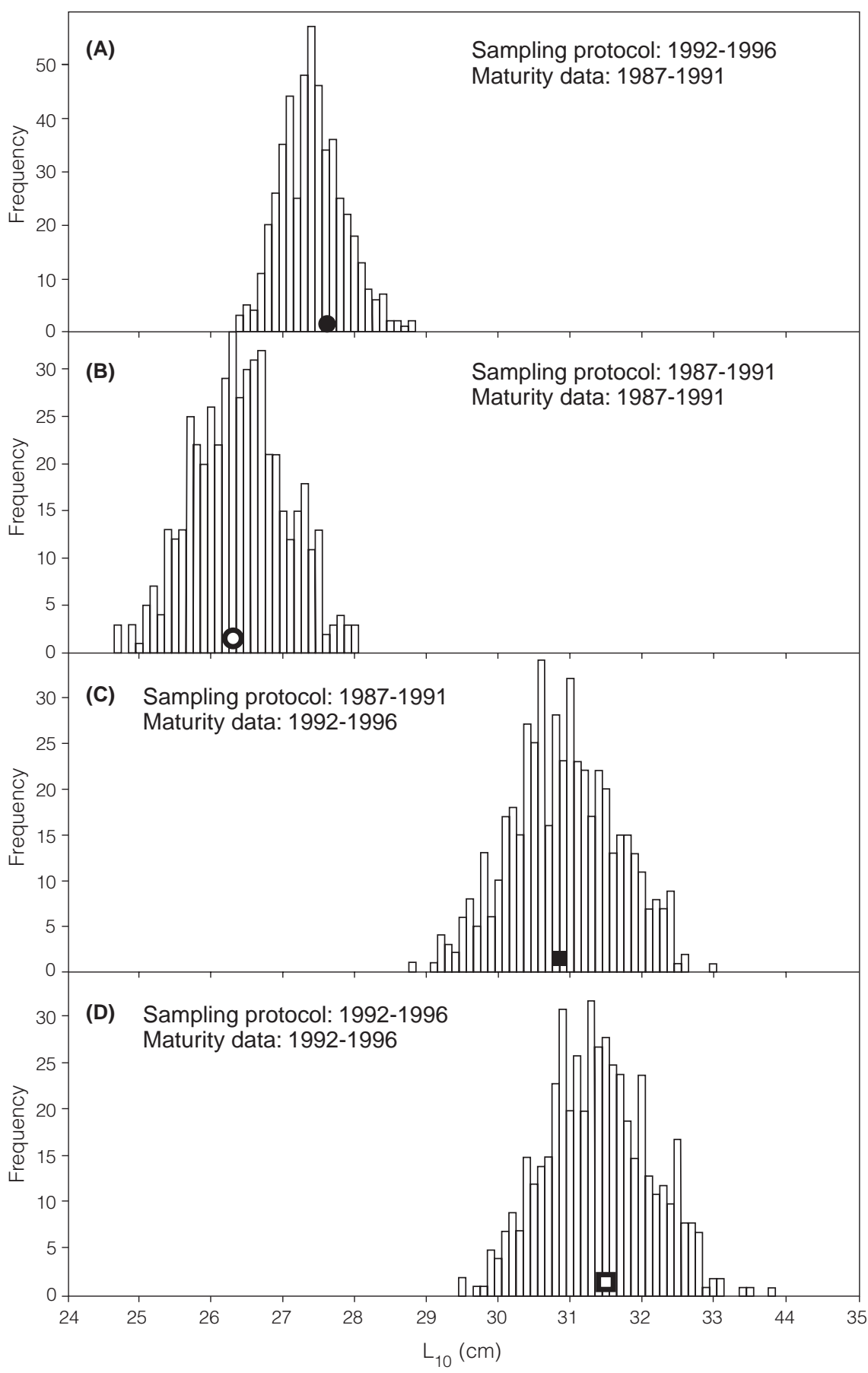

Fig. 5. Frequency distributions (bars) and medians (symbols) of estimated length at $10 \%$ maturity $\left(L_{10}\right)$ for female Georges Bank haddock obtained from 500 bootstrap replications of probit analyses for each of the four simulation scenarios: a) alternative sampling of 1987-91 data; b) baseline run for 1987-91; c) alternative sampling of 1992-96 data; and d) baseline run for 1992-96. 


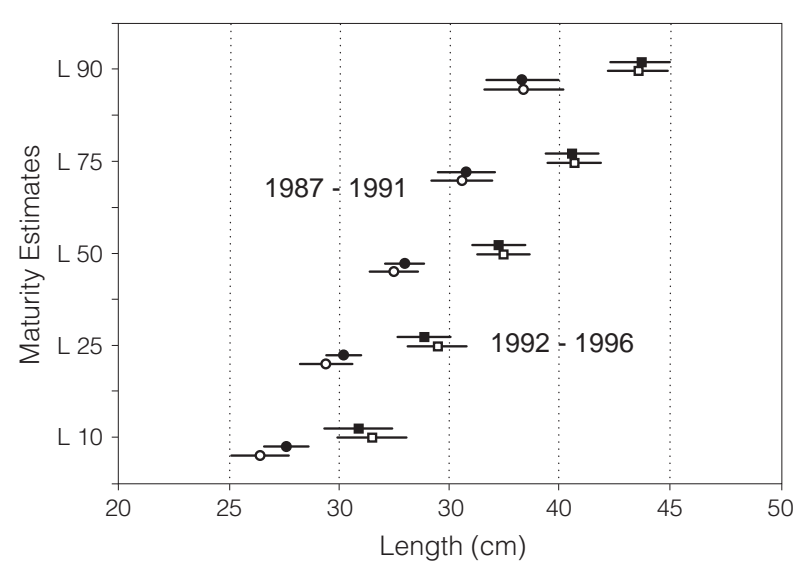

Fig. 6. Simulation results for Georges Bank haddock at levels of $L_{10}, L_{25}, L_{50}, L_{75}$ and $L_{90}$ for the four scenarios encompassing the periods 198791 and 1992-96; horizontal bars represent the $80 \%$ confidence interval of the frequency distributions associated with each estimate (see Fig. 6), symbols are the median values. Alternative sampling (closed symbol) results are positioned above baseline runs (open symbol) for each time period (circles represent estimates for the 1987-91, squares represent estimates for 1992-96).

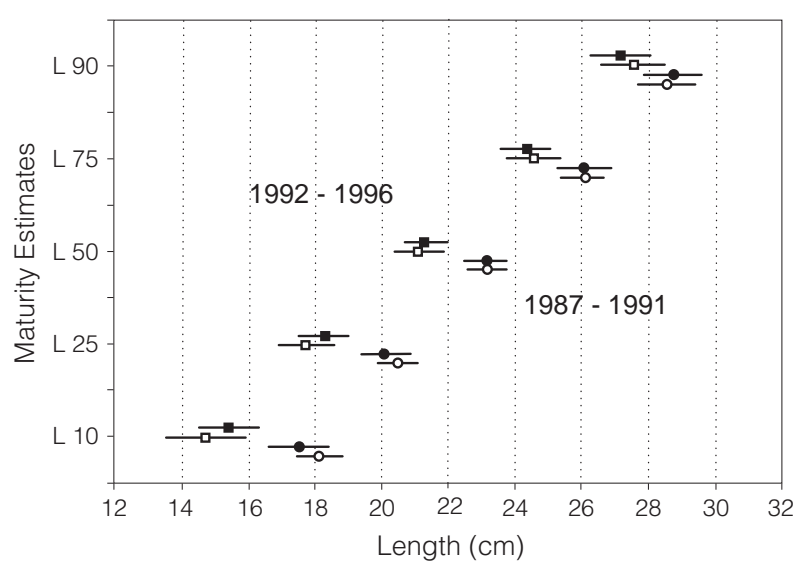

Fig. 7. Simulation results for male American plaice at levels of $L_{10}, L_{25}, L_{50}, L_{75}$ and $L_{90}$ for the four scenarios encompassing the periods 1987-91 and 1992-96; horizontal bars represent the $80 \%$ confidence interval of the frequency distributions associated with each estimate (see Fig. 6), symbols are the median values. Alternative sampling (closed symbol) results are positioned above baseline runs (open symbol) for each time period (circles represent estimates for the 1987-91, squares represent estimates for 1992-96).

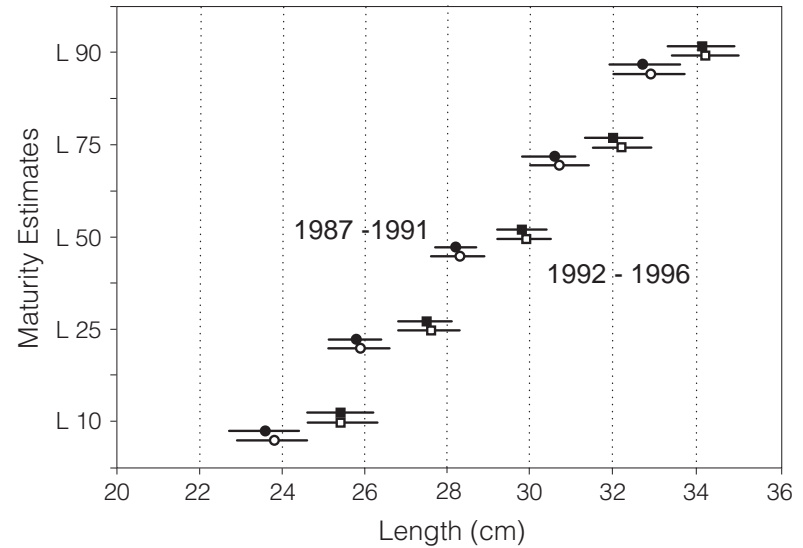

Fig. 8. Simulation results for male American plaice at levels of $L_{10}, L_{25}, L_{50}, L_{75}$ and $L_{90}$ for the four scenarios encompassing the periods 1987-91 and 1992-96; horizontal bars represent the $80 \%$ confidence interval of the frequency distributions associated with each estimate (see Fig. 6), symbols are the median values. Alternative sampling (closed symbol) results are positioned above baseline runs (open symbol) for each time period (circles represent estimates for the 1987-91, squares represent estimates for 1992-96).

1992-96 period. This indicates that the change in sampling scheme had no effect upon the maturity estimates, and that the difference in maturation rates between the two time periods is real and not an artifact. Similar results and conclusions were obtained for male and female American plaice (Fig. 7 and 8, respectively).

\section{Discussion}

The change in sampling scheme in 1992 resulted in significantly different length distributions of sampled fish after the change compared to 198791 period. Results from the Monte Carlo-type simulations indicate no bias was introduced in deriving maturity parameters for Georges Bank haddock and American plaice as a result of this change. However, simulation results did identify areas in which improved data collection might increase the precision of maturity analyses. Increased variability in simulation estimates for $L_{10}$ and $L_{90}$ (the lower and upper tails of the maturity ogive) suggests that additional sampling beyond that provided by lengthstratified age sampling may provide better estimates for this portion of the ogive. Estimates of $L_{50}$ 


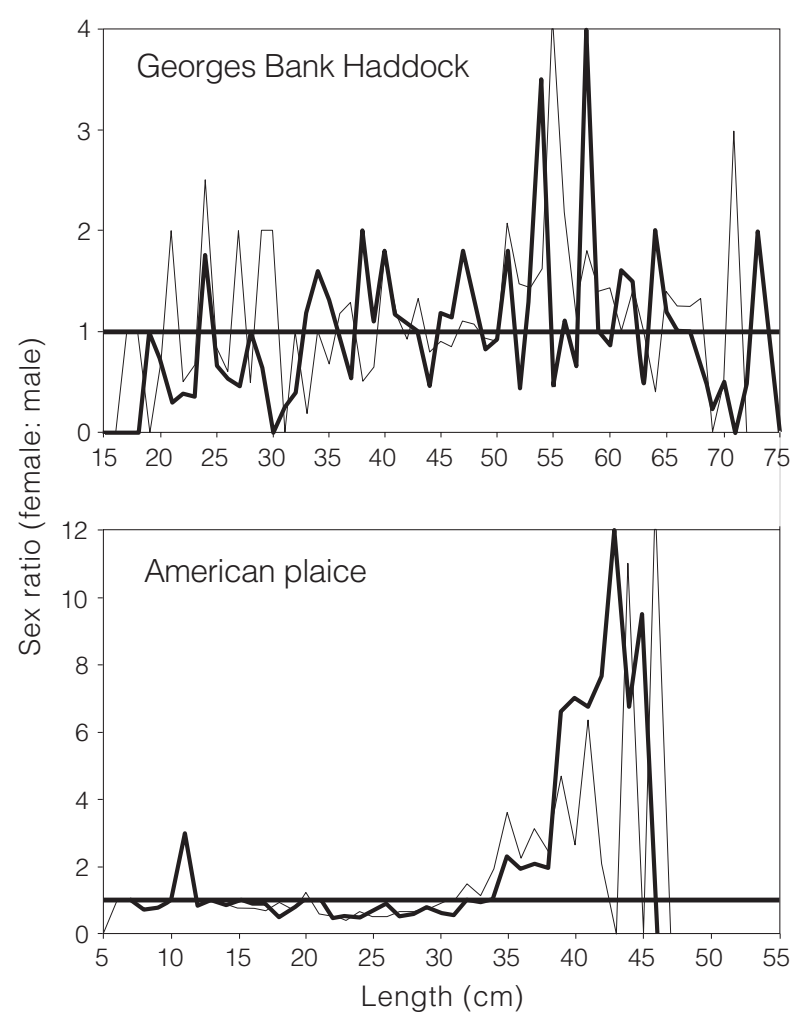

Fig. 9. Sex ratios (female:male) of Georges Bank haddock and American plaice sampled during NEFSC spring research vessel bottom trawl surveys, 1987-91 (thin line) and 1992-96 (thick line). Horizontal line denotes a 1:1 ratio.

appeared to be extremely robust with respect to the sampling scheme used, suggesting that this important parameter is well-established in the population and less susceptible to the vagaries of sampling than the upper and lower tail regions.

This study did not address the effect of lengthstratified age sampling on maturity at age (see Morgan and Hoenig, 1997), or the sex ratios of sampled fish effecting maturity parameters. In both the pre-1992 and post-1992 NEFSC sampling schemes, the sex of an individual fish is not determined until after the fish has been selected for sampling. In this study, results for haddock were prob- ably not affected since sex ratios for most gadoid species are 1:1 across the size range, and growth rates are similar for each sex. However, for American plaice, sex ratios became severely skewed towards females above $30 \mathrm{~cm}$ (Fig. 9), suggesting that for species exhibiting sexually dimorphic growth and maturation rates sampling modifications should be made. Causes for the different responses between male and female American plaice are unknown and pose interesting scientific issues. Results of this study, however, are sufficient to reject the hypothesis that changes in sampling protocols are responsible for the shifts.

\section{Acknowledgements}

We thank all the scientific staff involved in the collection of NEFSC research vessel survey data, and F. M. Serchuk, S.A. Murawski and two anonymous reviewers for their helpful comments.

\section{References}

ANON. MS 1994. Report of the workshop on sampling strategies for age and maturity. ICES C.M. Doc., No. D: $1,67 \mathrm{p}$.

ARMSTRONG, D. W., and S. ILARDIA. MS 1986. On bias in estimates of population parameters obtained by means of length-stratified sampling for age/length keys. ICES C.M. Doc., No. G:10, 17 p.

FINNEY, D. J. 1971. Probit analysis. $3^{\text {rd }}$ ed., Cambridge University Press, $333 \mathrm{p}$.

HALLIDAY, R. G. 1987. Size and age at sexual maturity of Atlantic argentine, Argentina silus: a critique. Env. Biol. Fishes, 19(2): 139-147.

MORGAN, M. J., and J. M. HOENIG. 1997. Estimating maturity-at-age from length stratified sampling. J. Northw. Atl. Fish. Sci., 21: 51-63.

NORTHEAST FISHERIES SCIENCE CENTER. MS 1992. Report of the thirteenth Northeast Regional Stock Assessment Workshop. NEFSC Ref. Doc., No. 92-02, 183 p.

O'BRIEN, L., J. BURNETT, and R. K. MAYO. 1993. Maturation of nineteen species of finfish off the Northeast coast of the United States, 1985-1990. NOAA Tech. Rep., NMFS, 113, 66 p.

SOKAL, R. R., and F. J. ROHLF. 1981. Biometry ( $2^{\text {nd }}$ edition), W. H. Freeman and Co., San Francisco, CA. 859 p. 